Aus aktuellem Anlass

Christina Anger* und Axel Plünnecke*

\title{
Schulische Bildung zu Zeiten der Corona-Krise
}

https://doi.org/10.1515/pwp-2020-0055

Zusammenfassung: Zur Eindämmung der Corona-Pandemie wurden die Schulen in Deutschland und in vielen anderen Ländern im März geschlossen. Homeschooling sollte verhindern, dass die Leistungen der Schülerinnen und Schüler abnehmen und die Ungleichheit der Bildungsleistungen steigt. Fehlende Konzepte und Erfahrungen der Lehrkräfte mit digitalem Fernunterricht sowie Ausstattungsmängel ließen jedoch vermuten, dass größere Lerndefizite entstanden sein dürften. Eine erste Studie aus Belgien bestätigt diese Befürchtungen. Die Ergebnisse dürften sich auf Deutschland übertragen lassen, denn auch hier bestehen erhebliche Defizite an digitalgestützten Lehrkonzepten. Um Vorsorge für den Fall neuerlicher Schulschließungen $\mathrm{zu}$ treffen und die Lücken zumindest in Teilen wieder zu verringern, sollte man die Chancen der Digitalisierung zur Verbesserung des Unterrichts nutzen. Erste Impulse hat es bereits gegeben: Die Ausstattung mit digitalen Endgeräten bessert sich, Lehrplattformen stehen zur Verfügung, digitale Bildungsinhalte werden entwickelt. Auch der WLAN-Zugang an den Schulen soll in den kommenden Jahren im Zuge der praktischen Umsetzung des Digitalpakts verbessert werden. Als nächste Schritte gilt es die Lehrkräfte weiterzubilden, FeedbackMechanismen zum Unterricht zu schaffen, die Lehrkräfte durch IT-Support zu unterstützen und Lerninhalte auf den Plattformen motivierend und effektiv darzureichen, begleitet durch wissenschaftliche Evaluation.

JEL-Klassifikation: I21, I24

Schlüsselwörter: Bildungsökonomie, Digitalpakt, Digitalisierung, Schulschließungen

*Kontaktpersonen: Christina Anger, Institut der deutschen Wirtschaft, Kompetenzfeld Bildung, Zuwanderung und Innovation, Konrad-Adenauer-Ufer 21, 50668 Köln, E-Mail: anger@iwkoeln.de Axel Plünnecke, Institut der deutschen Wirtschaft, Kompetenzfeld Bildung, Zuwanderung und Innovation, Konrad-Adenauer-Ufer 21, 50668 Köln, E-Mail: pluennecke@iwkoeln.de

\section{Herausforderungen der Schulen seit Beginn der Corona-Krise}

Um die Ausbreitung des Corona-Virus zu verlangsamen, wurden die Schulen in Deutschland und in vielen anderen Ländern ab Mitte März für etwa zwei Monate geschlossen. Normaler Unterricht fand in dieser Zeit nicht statt. In der Regel stellten Lehrkräfte den Schülerinnen und Schülern Unterrichtsmaterialien zur Verfügung und boten via Telefon oder moderne digitale Kommunikationswege Hilfestellungen an. Seit Anfang bis Mitte Mai kehrten die Bundesländer schrittweise zum Präsenzunterricht zurück. Dabei fand dieser in vielen Fällen nur tageweise und mit verringertem Zeitumfang statt; an den anderen Tagen gab es weiterhin Fernunterricht. Nach den Sommerferien sind alle Bundesländer wieder zu einem Regelbetrieb in den Schulen mit bestimmten Hygieneplänen und anderen Restriktionen übergegangen. Bisher gab es aufgrund von Infektionen der Lehrkräfte oder von Schülerinnen und Schülern nur vereinzelt wieder temporäre Schulschließungen oder einzelne Klassen wurden vorübergehend wieder vom Präsenzunterricht ausgeschlossen (Stand: 22. Oktober 2020). Die Politik hält daher weiter am Regelbetrieb fest, versucht parallel dazu Vorkehrungen für den Fall eines neuerlich temporären und regional notwendigen digitalen Fernunterrichts $\mathrm{zu}$ treffen und die Infrastruktur in den Schulen zu verbessern.

Die Reaktionen der Bildungspolitik auf die CoronaKrise bringen die Frage auf, wie groß der Effekt der Schulschließungen auf die Bildungsleistungen der Schülerinnen und Schüler tatsächlich ist und welche Auswirkungen es im Hinblick auf Bildungsgerechtigkeit und Bildungsarmut gibt. Wie stark konnte man diese Effekte durch Homeschooling in der ersten Phase der Corona-Krise abpuffern? Welche Entwicklungen gibt es seitdem in der Digitalisierung der Schulen? Und welche Handlungsempfehlungen lassen sich zum gegenwärtigen Zeitpunkt ableiten? 


\section{Effekte von Schulschließungen auf die Bildungsleistungen der Schülerinnen und Schüler}

Um den Effekt der Schulschließungen zu ermitteln, kann man auf die Berechnung von Auswirkungen früherer Schulschließungen oder Lernunterbrechungen zurückgreifen. Eine Obergrenze lässt sich aus empirischen Untersuchungen zu Schulstreiks ableiten. Ein halbjähriger Lehrerstreik 1990 im französischen Teil Belgiens beispielsweise führte dazu, dass sich die Wahrscheinlichkeit von Klassenwiederholungen erhöhte und dass die Schüler im Durchschnitt niedrigere Schulabschlüsse erreichten (Belot und Webbink 2010). Streiks chilenischer Studenten und Schüler 2011 führten dazu, dass sich die Leistungen in Mathematik verschlechterten und auch die Übergangsquoten an den Universitäten abnahmen. Die negativen Effekte sind für einen längeren Zeitraum nachweisbar (Gaete 2018). Langfristig negative Effekte zeigen Jaume und Willén (2019) anhand von Daten für Argentinien. Ein längerer Unterrichtsausfall während der Grundschulzeit kann bei den Betroffenen noch im Alter von 30 bis 40 nachgewiesen werden. Das durchschnittliche Arbeitseinkommen ist niedriger und die Gefahr von Arbeitslosigkeit größer. Besonders stark sind die negativen Auswirkungen der Schulschließungen für Kinder aus bildungsfernen Haushalten und von Eltern mit geringeren Einkommen. Daher stellen Schulschließungen vor allem für das Ziel der Bildungsgerechtigkeit ein besonderes Problem dar.

Auch Studien zum „Summer gap“ zeigen, dass Kinder aus bildungsfernen Haushalten durch den fehlenden Unterricht im Vergleich zu anderen Kindern stark zurückfallen (Karl et al. 2007). Kuhfeld und Tarasawa (2020) übertragen die Effekte des Summer gap in Projektionsmodellen auf die Corona-bedingten Schulschließungen und finden besonders starke Einbrüche in den mathematischen Kompetenzen, die wiederum umso größer ausfallen, je jünger die Schülerinnen und Schüler sind.

Kinder mit Migrationshintergrund dürften besonders stark von den Auswirkungen der Schulschließungen betroffen sein. Ein Teil dieser Kinder profitiert stark von der Sprachförderung in Kitas und Grundschulen. Besondere Potenziale bieten dabei Ganztagseinrichtungen (Anger und Geis-Thöne 2018). Wenn aber die Schulen geschlossen sind und keine Fördermöglichkeiten bestehen, drohen Kinder mit Migrationshintergrund besonders stark zurückzufallen. Im Normalfall können Schulsozialarbeit, Vernetzung aller Verantwortlichen vor Ort, breite Beratungsangebote sowie Maßnahmen für die einzelnen Jugendlichen Schulabbrüche vermeiden (Liessem 2015). Solche Präven- tionsprogramme können langfristig positive Auswirkungen auf Einkommen und Beschäftigung haben und die Wahrscheinlichkeit verringern, dass die Kinder später im Leben auf soziale Leistungen angewiesen sind (Lavecchia et al. 2019). Doch während des Lockdowns waren sowohl die Schulen als auch andere wichtige Akteure vor Ort in ihrer präventiven Arbeit stark eingeschränkt. Das bedeutet, dass die Zahl der Schulabbrüche zunehmen könnte.

Di Pietro et al. (2020) leiten aus der bestehenden Literatur zu Schulunterbrechungen vor der Corona-Krise zusammenfassend ab, dass pro Woche der Schulschließung infolge der Corona-Krise ein Verlust der Lernleistung von 0,8 bis 2,3 Prozent der Standardabweichung zu erwarten ist. Für eine Schulunterbrechung von 10 Wochen wären damit durchschnittliche Lernverluste von bis zu 23 Prozent der Standardabweichung zu erwarten.

\section{Kompensation der potenziellen negativen Effekte durch Homeschooling}

Die Literatur zu Schulstreiks und zum Summer gap erlaubt nur die empirische Abschätzung einer Obergrenze der negativen Effekte der Corona-bedingten Schulschließungen, da sich diese nur auf den Präsenzunterricht bezogen haben. Ab Mitte März trat jedoch eine Form von Homeschooling an die Stelle des Präsenzunterrichts, wobei die Lehrkräfte vor allem auf Aufgaben zurückgegriffen haben, die Schülerinnen und Schüler selbstreguliert erledigen. Hierfür brauchen diese jedoch Motivation und Kompetenzen wie Selbststeuerung, die überhaupt erst durch das Bildungssystem vermittelt werden müssen (Ramdass und Zimmerman 2011). Man kann nicht davon ausgehen, dass diese Kompetenzen zuvor schon in ausreichendem Maße von den Schulen vermittelt worden waren. Insbesondere jüngere Kinder in der Grundschule und in weiterführenden Schulen dürften Unterstützung benötigt haben.

Eine Elternbefragung von Wößmann et al. (2020) zeigt, dass Schulkinder ihre Zeit für schulische Aktivitäten mehr als halbierten, von durchschnittlich 7,4 Stunden vor der Corona-Krise auf 3,6 Stunden während der CoronaKrise. Während dabei die schulbezogenen Lernzeiten zwischen Akademiker- und Nichtakademikerkindern nicht auseinanderklafften, gab es große Unterschiede zwischen leistungsstärkeren und leistungsschwächeren Kindern. Besonders Schülerinnen und Schüler, die bereits vor der Corona-Krise größere Lernschwierigkeiten hatten, schränkten ihre Lernzeiten stärker ein (Wößmann et al. 2020). Ähnliche Effekte treten selbst bei älteren Schüle- 
KS 1: Mutter ohne beruflichen Abschluss KS 1: Vater ohne beruflichen Abschluss KS 1: Mutter mit Berufsausbildung

KS 1: Vater mit Berufsausbildung KS 1: Mutter mit Hochschulabschluss KS 1: Vater mit Hochschulabschluss

KS 2: Mutter ohne beruflichen Abschluss

KS 2: Vater ohne beruflichen Abschluss KS 2: Mutter mit Berufsausbildung

KS 2: Vater mit Berufsausbildung KS 2: Mutter mit Hochschulabschluss KS 2: Vater mit Hochschulabschluss

KS 3: Mutter ohne beruflichen Abschluss KS 3: Vater ohne beruflichen Abschluss KS 3: Mutter mit Berufsausbildung

KS 3: Vater mit Berufsausbildung KS 3: Mutter mit Hochschulabschluss KS 3: Vater mit Hochschulabschluss

KS 4: Mutter ohne beruflichen Abschluss KS 4: Vater ohne beruflichen Abschluss KS 4: Mutter mit Berufsausbildung

KS 4: Vater mit Berufsausbildung KS 4: Mutter mit Hochschulabschluss

KS 4: Vater mit Hochschulabschluss

KS 5/6: Mutter ohne beruflichen Abschluss KS 5/6: Vater ohne beruflichen Abschluss KS 5/6: Mutter mit Berufsausbildung

KS 5/6: Vater mit Berufsausbildung KS 5/6: Mutter mit Hochschulabschluss KS 5/6: Vater mit Hochschulabschluss

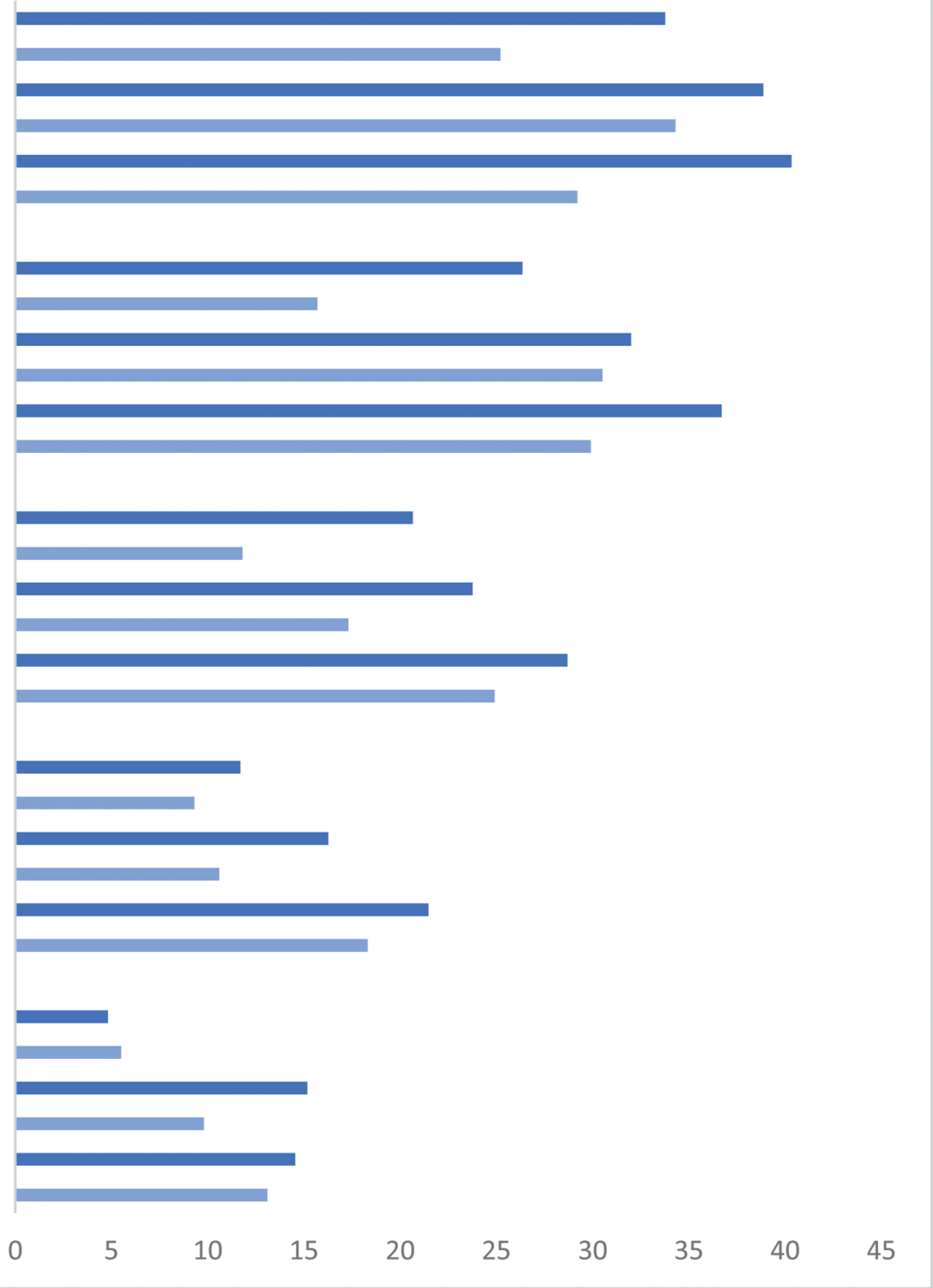

Abbildung 1: Unterstützung durch die Eltern bei den Hausaufgaben in Abhängigkeit vom Bildungshintergrund der Eltern und den Kompetenzstufen der Kinder bei PISA

Anmerkung: Anteil der Mütter bzw. Väter, die ihre Kinder mehrmals im Monat oder mehrmals in der Woche bei den Hausaufgaben unterstützen, in Prozent; KS: Kompetenzstufe bei PISA.

Quelle: Anger und Plünnecke 2020a

rinnen und Schülern am Gymnasium auf (S. Anger et al. 2020). Eine Befragung von Oberstufenschülerinnen und -schülern zeigt, dass nur 37 Prozent während des Homeschoolings mehr als 2 Stunden am Tag mit Lernen für die Schule verbrachten. Schülerinnen und Schüler mit schlechteren Noten waren weniger aktiv.

Auch die Eltern spielen während der Corona-Krise beim Homeschooling eine wichtige Rolle. Doepke und Zilibotti (2019) betonen, dass die an Zeit und Qualität gemessen unterschiedliche Förderung durch Eltern mit unterschiedlichen Bildungsressourcen eine zentrale Ursache der Ungleichheit der Bildungschancen ist. Den Zusam- menhang zwischen dem Bildungshintergrund der Eltern und ihrer Unterstützung der Kinder zeigen auch Anger und Plünnecke (2020a) anhand von Auswertungen der PISADaten aus dem Jahr 2018. Abbildung 1 verdeutlicht, dass Eltern aus Akademikerhaushalten mehr Zeit für die Unterstützung der Kinder beim schulischen Lernen aufwenden - und dies unabhängig von dem Kompetenzniveau der Kinder im PISA-Test.

Ein weiterer Einfluss des Elternhauses auf den Bildungserfolg während der Phase des Homeschoolings geht auch von unterschiedlichen räumlichen und infrastrukturellen Bedingungen aus. Kinder aus bildungsfernen Haus- 
halten verfügen seltener über einen eigenen PC oder ein Tablet und auch deutlich seltener über einen ruhigen Arbeitsplatz. Basierend auf Auswertungen der SOEP-Daten aus dem Jahr 2018 besitzen 43 Prozent aller Zwölfjährigen und 52 Prozent aller Vierzehnjährigen einen eigenen Computer oder ein Tablet. Für Kinder aus bildungsfernen Haushalten trifft dies nur auf 31 bzw. 34 Prozent zu (Geis-Thöne 2020).

Neben dem geringeren Zeitaufwand für das Lernen und den problematischen Lernvoraussetzungen ist auch die (noch) fehlende Qualität der Fernbeschulung zu bemängeln. Nach der Metastudie von Hattie (2013) ergibt sich eine hohe Unterrichtsqualität, wenn Lehrpersonen regelmäßig Feedback zu ihrem Unterricht einholen, gezielt an der Verbesserung ihres Verhaltens im Unterricht arbeiten und auch ihrerseits den Schülern Rückmeldungen geben. Hausaufgaben hingegen haben nur kleinere Effekte. Gerade das qualitätsstärkende Feedback an Schülerinnen und Schülern fand jedoch in der Phase des Fernunterrichts kaum statt, wie Befragungen von Lehrkräften deutlich machen (Hachfeld et al. 2020). Auch konnten die Lehrkräfte nicht vom Feedback anderer Lehrkräfte zu ihrem digitalen Fernunterricht profitieren.

Erste Auswertungen zu Lernerfolgen während der Corona-Krise kommen daher auch zu ernüchternden Ergebnissen. Daten zur Nutzung des Online-Mathematik-Programms Zearn in den Vereinigten Staaten zeigen, dass der Lernfortschritt der Schülerinnen und Schüler während der Corona-Krise einbrach. Insbesondere die Leistungen von Kindern aus sozioökonomisch benachteiligten Familien gingen deutlich zurück und blieben über den gesamten Zeitraum der Schulschließungen gering (Chetty et al. 2020).

Eine erste Untersuchung zu den Effekten der Schulschließungen auf in Leistungstests gemessene Kompetenzen während der Corona-Krise liegt inzwischen für Flandern in Belgien vor (Maldonado und De Witte 2020). In Flandern waren die Schulen ohne Vorbereitung vom 16. März bis zum 17. Mai geschlossen, für neun Wochen inklusive der Osterferien. Danach öffneten die Grund- und weiterführenden Schulen schrittweise wieder. In den drei Wochen vor den Osterferien haben die Schülerinnen und Schüler zumeist bestehendes Lernmaterial und Aufgaben im Homeschooling wiederholt. In einigen Grundschulen wurden Lernplattformen eingesetzt und Aufgaben online zur Verfügung gestellt. Dabei gab es aber Probleme, weil digitale Endgeräte fehlten. In der Phase nach den Osterferien wurde ein Fernunterricht konzipiert, der sich für die Schülerinnen und Schüler über etwa vier Stunden pro Tag erstrecken sollte. Ab dem 18. Mai 2020 begann der Präsenzunterricht für die 6. Jahrgangsstufe an maximal zwei
Tagen pro Woche. An den anderen Tagen fand weiterhin Distanzunterricht statt. Ab dem 8. Juni öffneten die Schulen wieder voll (Moldonado und De Witte 2020). Dauer und Umfang der Schulschließungen sind damit annähernd mit den Maßnahmen in vielen Bundesländern in Deutschland vergleichbar.

Moldonado und De Witte (2020) zeigen auf Basis der standardisierten Testergebnisse aus dem Juni 2020 für die Sechstklässler, dass diese im Vergleich zu früheren Kohorten einen Lernverlust von 0,19 Standardabweichungen in Mathematik und 0,29 Standardabweichungen in Niederländisch aufweisen. Zugleich hat die Ungleichheit stark zugenommen - sowohl innerhalb als auch zwischen Schulen, wobei Schulen mit mehr benachteiligten Schülerinnen und Schülern größere Einbußen erlitten haben.

Ob die Effekte aus Belgien in gleicher Höhe in Deutschland eintreten, müssen weitere empirische Studien zeigen. Die Effekte lassen sich übertragen, falls der Fernunterricht in Deutschland nicht besser als in Belgien war. Langfristig ist es entscheidend, ob es gelingt, die entstandenen Defizite in den kommenden Jahren durch eine Weiterentwicklung des Bildungssystems wieder zu einem Teil zu beheben.

\section{Digitale Ausgangslage der Schulen}

Gemäß der International Computer and Information Literacy Study (ICILS) war die Ausstattung der Schulen in Deutschland mit digitalen Geräten im Jahr 2018 deutlich schlechter als im internationalen Durchschnitt. Nur 26,2 Prozent der Schülerinnen und Schüler in der achten Klasse besuchten 2018 eine Schule, in der sowohl für sie als auch für die Lehrkräfte ein WLAN-Zugang verfügbar war. Dänemark, das Land mit den höchsten computer- und informationsbezogenen Kompetenzen der Schülerinnen und Schüler, erreicht eine Quote von 100 Prozent. An den deutschen Schulen besteht für Achtklässler im Durchschnitt ein Schüler-Computer-Verhältnis von 9,7:1. In Dänemark ist das entsprechende Verhältnis 4,6:1. Auch die Lehrkräfte sind in Deutschland schlecht ausgestattet. Während 3,2 Prozent der Schülerinnen und Schüler in Deutschland Schulen besuchen, in denen alle Lehrkräfte tragbare digitale Endgeräte erhalten, liegt der entsprechende Anteil in Dänemark bei 91,1 Prozent. Internetbasierte Anwendungen für gemeinschaftliches Arbeiten von Lehrkräften und Schülerinnen und Schülern waren in Dänemark 2018 für 96,8 Prozent der Schülerpopulation vorhanden, in Deutschland nur für 16,5 Prozent (Eickelmann et al. 2019). 
In der PISA-Erhebung aus dem Jahr 2018 wurden die Schülerinnen und Schüler befragt, wie häufig digitale Geräte in verschiedenen Unterrichtsstunden Einsatz finden. Zwischen 6 und 8 Prozent der Neuntklässler in Deutschland setzen digitale Geräte mehr als 60 Minuten pro Woche im Unterricht in Deutsch, Mathematik oder Naturwissenschaften ein. Mit gut 8 Prozent ist die Nutzung in Belgien ähnlich gering. In Dänemark haben die Schülerinnen und Schüler mehr Erfahrung. Der Schüleranteil, der digitale Endgeräte mehr als eine Stunde pro Woche nutzt, liegt hier zwischen 55 Prozent in Naturwissenschaften und 77 Prozent in Dänisch (Tabelle 1).

Tabelle 1: Einsatz von digitalen Geräten in einer typischen Schulwoche in Prozent

Nie 1-30 Minuten 31-60 Minuten Mehrals 60

Minuten

\begin{tabular}{lllll}
\hline Deutschland & & & & \\
\hline Testsprache & 65,7 & 22,2 & 5,8 & 6,0 \\
\hline Mathematik & 64,5 & 19,4 & 8,1 & 7,6 \\
\hline $\begin{array}{l}\text { Naturwis- } \\
\text { senschaften }\end{array}$ & 52,9 & 28,0 & 11,4 & 6,3
\end{tabular}

senschaften

\begin{tabular}{lllll}
\hline Dänemark & & & & \\
\hline Testsprache & 1,6 & 7,1 & 14,1 & 76,8 \\
\hline Mathematik & 4,3 & 14,0 & 20,5 & 61,0 \\
\hline Naturwis- & 4,9 & 14,2 & 24,9 & 55,5 \\
senschaften & & & &
\end{tabular}

\begin{tabular}{lcccc}
\hline Belgien & & & & \\
\hline Testsprache & 49,9 & 19,0 & 8,2 & 8,0 \\
\hline Mathematik & 57,2 & 12,9 & 6,0 & 8,5 \\
\hline $\begin{array}{l}\text { Naturwis- } \\
\text { senschaften }\end{array}$ & 53,3 & 13,2 & 7,4 & 8,2 \\
\hline
\end{tabular}

Anmerkung: Die Angaben addieren sich nicht zu 100, weil einige Schülerinnen und Schüler das jeweilige Fach nicht belegt haben. Quelle: Eigene Berechnungen auf Basis PISA 2018, Befragung von Neuntklässlern

Bei den Angaben zum Einsatz digitaler Endgeräte zeigen sich ebenso wichtige Unterschiede. Eine gemeinsame Nutzung digitaler Geräte im Unterricht von Lehrkräften sowie Schülerinnen und Schülern berichtet rund ein Fünftel der Neuntklässler in Deutschland. Ähnliche Erfahrungen weisen die Neuntklässler in Belgien auf. In Dänemark hingegen haben 77 Prozent der Neuntklässler in Naturwissenschaften und sogar 89 Prozent der Neuntklässler in Dänisch digitale Geräte im Unterricht gemeinsam mit Lehrkräften genutzt (Tabelle 2).
Tabelle 2: Einsatz von digitalen Geräten im Schulunterricht während des letzten Monats nach Nutzer in Prozent

\begin{tabular}{|c|c|c|c|}
\hline $\begin{array}{r}\text { Nutzung } \\
\text { durch Lehrer } \\
\text { und Schüler }\end{array}$ & $\begin{array}{l}\text { Nutzung nur } \\
\text { durch Schüler }\end{array}$ & $\begin{array}{l}\text { Nutzung nur } \\
\text { durch Lehrer }\end{array}$ & $\begin{array}{r}\text { Keine } \\
\text { Nutzung }\end{array}$ \\
\hline
\end{tabular}

\begin{tabular}{lcccc}
\hline Deutschland & & & & \\
\hline Testsprache & 20,0 & 9,8 & 19,3 & 50,2 \\
\hline Mathematik & 20,8 & 9,0 & 17,4 & 51,9 \\
\hline $\begin{array}{l}\text { Naturwis- } \\
\text { senschaften }\end{array}$ & 21,9 & 11,2 & 27,1 & 37,8 \\
\hline
\end{tabular}

senschafte

\begin{tabular}{lcccc}
\hline Dänemark & & & & \\
\hline Testsprache & 88,9 & 6,9 & 2,7 & 1,2 \\
\hline Mathematik & 80,3 & 11,1 & 4,4 & 3,9 \\
\hline $\begin{array}{l}\text { Naturwis- } \\
\text { senschaften }\end{array}$ & 77,3 & 11,9 & 6,6 & 3,5
\end{tabular}

\begin{tabular}{lllll}
\hline Belgien & & & & \\
\hline Testsprache & 23,5 & 7,3 & 32,0 & 18,7 \\
\hline Mathematik & 15,9 & 6,5 & 34,0 & 23,9 \\
\hline $\begin{array}{l}\text { Naturwis- } \\
\text { senschaften }\end{array}$ & 14,5 & 5,9 & 36,1 & 20,4 \\
\hline
\end{tabular}

Anmerkung: Die Angaben addieren sich nicht zu 100, da einige Schülerinnen und Schüler das jeweilige Fach nicht belegt haben. Quelle: Eigene Berechnungen auf Basis PISA 2018, Befragung von Neuntklässlern

Eine aktuelle Auswertung der PISA-2018-Daten durch die OECD (2020) macht deutlich, dass Deutschland in den betrachteten Indikatoren (Verfügbarkeit einer OnlineLernplattform, entsprechende Ressourcen zur Weiterbildung für Lehrkräfte) deutlich schlechter abschneidet als der OECD-Durchschnitt. Auch bei diesen beiden Indikatoren schneidet Dänemark besser als der OECD-Durchschnitt ab, Belgien weist Werte nah am OECD-Durchschnitt auf.

Schon vor etwa 20 Jahren hat Dänemark mit der Digitalisierung der Schulen begonnen. Neben der Infrastruktur (WLAN, Computerausstattungen) wurde auch in Lernplattformen und Software zum gemeinschaftlichen Arbeiten investiert (Leopoldina 2020). Zudem nehmen Lehrkräfte in Dänemark stärker an Weiterbildungen teil und geben einander mehr Feedback (Eickelmann et al. 2019). Dadurch konnte Dänemark die Erfahrungen der Lehrkräfte sowie der Schülerinnen und Schüler mit digitalem Unterricht nutzen und war in der Lage, zu Beginn der Corona-Krise die Schulen schnell zu schließen und den Unterricht digital weiterzuführen. Bereits nach wenigen Wochen öffnete Dänemark die Schulen wieder. Um Abstandsregelungen einzuhalten, nutzte man auch Wechselmodelle zwischen Präsenz- und Distanzlernen (Leopoldina 2020). 
Die Digitalisierung der Schulen hilft nicht nur in der Corona-Krise, wenn es darum geht, von Präsenzunterricht auf Distanzunterricht zu wechseln und dabei Qualitätsverluste im Unterricht $\mathrm{zu}$ vermeiden. Auch für den Regelbetrieb kann sie positive Effekte erzeugen. Untersuchungen von Falck et al. (2018) zeigen jedoch auf Basis der TIMMS-Erhebung aus dem Jahr 2011, dass der Einsatz von Computern im Unterricht nicht für alle Einsatzbereiche positive Effekte erzeugt und dass ein zu breiter Einsatz $\mathrm{zu}$ keiner Qualitätsverbesserung führt. Hillmayr et al. (2017) kommen auf Basis einer Metastudie zum Einsatz digitaler Medien zu dem Schluss, dass digitale Methoden die Leistungen der Schülerinnen und Schüler erhöhen. Der positive Effekt steigert sich, wenn digitale mit traditionellen Methoden kombiniert und die Lehrkräfte entsprechend qualifiziert werden. Die ICILS-Studie zeigt jedoch, dass sich 2018 nur wenige Lehrkräfte in Deutschland für den Einsatz digitaler Medien weitergebildet haben und dass nur 5,7 Prozent der Achtklässler Schulen besuchten, in denen viele Lehrkräfte Unterrichtshospitationen zum Einsatz digitaler Medien machten. Nur 34,7 Prozent der Lehrkräfte in Deutschland gaben an, dass der schulische Einsatz digitaler Medien die Leistungen der Schülerinnen und Schüler verbessert. In Dänemark betrug der entsprechende Anteil 74,8 Prozent (Eickelmann et al. 2019).

\section{Erste Schritte der Transformation der Schulen}

Auf der Suche nach Handlungsempfehlungen dafür, wie sich die Herausforderungen der Corona-Krise meistern lassen, sind zwei Schwerpunkte zu unterscheiden. Zum einen sollten die Schulen während des weiteren Verlaufs der Pandemie mit hoher Priorität offen gehalten und dazu die Bedingungen an den Schulen verbessert werden. Zum anderen sollte die Qualität der Schulen im Regelbetrieb erhöht werden. In beiden Handlungsfeldern kommt der weiteren Digitalisierung eine besondere Bedeutung zu.

Um die Schulen offen zu halten, sind die allgemeinen Regeln zur Eindämmung der Pandemie auf die Schulen zu übertragen. Alltagsmasken können auf den Schulwegen sowie auf Wegen in Schulgebäuden helfen, wenn sich dort keine sicheren Abstände einhalten lassen. Insbesondere die Gewährleistung von Hygienemaßnahmen wurde im Vorfeld der Schulöffnungen mit hoher Priorität in die Praxis umgesetzt. Im Klassenzimmer selbst sind Abstände, Lüftung und Masken wichtig. In Klassenräumen, in denen diese Konzepte nicht umsetzbar sind, sollte hochwertige Lüftungstechnik eingesetzt werden. In den Schulen wird ferner darauf geachtet, dass möglichst keine Mischung der Gruppen ohne Maske und Abstand eintritt. Einige Konzepte der Bundesländer sehen für Kreise und Städte nach wöchentlichen Neuinfektionszahlen differenzierte Pläne zum Einsatz von Masken und einer Teilung der Klassen durch hybride Lehrformate vor (Anger und Plünnecke 2020b). Für Schulklassen, in denen ein Kind positiv auf das Corona-Virus getestet wurde, wird als Vorsichtsmaßnahme vom Gesundheitsamt oftmals eine Quarantäne angeordnet. Für diese Klassen findet dann wiederum für kurze Zeit Fernunterrichtet statt. Ende September 2020 galt dies für deutlich weniger als ein Prozent der Schülerschaft.

Für den Fall des Fernunterrichts und für die Umsetzbarkeit der hybriden Lehrformate spielt die weitere Digitalisierung eine zentrale Rolle. Wie oben beschrieben, schätzten die Lehrkräfte 2018 den Nutzen der Digitalisierung als eher gering ein. Für den Erfolg der Transformation war es wichtig, dass mit den Schulschließungen die Notwendigkeit und Dringlichkeit des Wandels erkannt wurde. Auch wurde seit den Schulschließungen die Bedeutung einer Vision und Konzeption erkannt, und die Unterstützung für den Transformationsprozess bei den Lehrkräften steigt (Anger und Plünnecke 2020b).

Zudem werden seit dem Sommer die Bemühungen verstärkt, die Voraussetzungen für digitalen Unterricht zu verbessern. So wurden beispielsweise zusätzliche finanzielle Mittel des Bundes bereitgestellt, um mehr Leihgeräte für Schülerinnen und Schüler und Dienstgeräte für Lehrkräfte zur Verfügung zu stellen. Auch die WLAN-Verfügbarkeit an den Schulen soll verbessert werden. Darüber hinaus dürften in der nächsten Zeit verstärkt Mittel aus dem Digitalpakt abgerufen werden, da inzwischen die Notwendigkeit einer weiteren Digitalisierung der Schulen ausreichend erkannt ist. Bis zum Frühjahr 2020 wurden erst wenige Anträge für Mittel aus dem Digitalpakt gestellt. Zudem verfügen inzwischen alle Bundesländer über Lernplattformen, und nach einem Koalitionsbeschluss von Ende August 2020 soll eine Bildungsplattform mit digitalen Lerninhalten für ganz Deutschland entwickelt werden.

Um den Transformationsprozess weiter voran zu bringen, ist es ebenso wichtig, die praktische Umsetzung der Digitalisierungsstrategie zu unterstützen. Mit $20.000 \mathrm{zu}$ sätzlichen IT-Stellen an den Schulen könnte man die Administration sicherstellen und die Lehrkräfte unterstützen (C. Anger et al. 2020). Dies würde nicht nur dazu dienen, die Digitalisierungsstrategie umzusetzen, sondern es würde auch künftige strategische Überlegungen an den Schulen beeinflussen. Als zweites sind die Lehrkräfte für den Einsatz digitaler Technologien im Unterricht und für das 
Begleiten der Schülerinnen und Schüler im Homeschooling zu qualifizieren. Bisher haben nur wenige Bundesländer entsprechende Vorgaben für das Lehramtsstudium erlassen (Autorengruppe Bildungsberichterstattung 2020). Köller (2020, S. 15) fordert hierzu die „Integration der informations- und computerbezogenen Bildung in den berufswissenschaftlichen Anteil der ersten Phase der Lehrkräfteausbildung“. Um durch zusätzliche Fort- und Weiterbildungsangebote für digitale Lernformate die Qualität des Unterrichts zu erhöhen (Eickelmann und Drossel 2020), sind Zeiten für deren Nutzung sicherzustellen. Drittens sollte das Feedback der Lehrkräfte untereinander zum digitalen Unterricht weiter gesteigert werden. Wichtige Impulse zum Feedback und zur qualitativen Weiterentwicklung des Unterrichts sollten auch aus den kommenden Vergleichsarbeiten empirisch abgeleitet werden, in dem man diese so weiterentwickelt, dass sie erste digitale Unterrichtstrategien definieren und erfassen. Viertens sollte eine intelligente Lernsoftware entwickelt werden, die Schülerinnen und Schüler motiviert und Lerndefizite beheben kann. Montag et al. (2019) beschreiben verschiedene psychologische Mechanismen, die sich zur Motivation der Schülerinnen und Schüler nutzen lassen. Nach Empfehlung von Köller (2020) ist die schnelle Einrichtung eines Gremiums mit Vertretern aus Politik, Schulbuchverlagen, kommerzieller Softwareentwicklung und Wissenschaft aus den Bereichen KI und Lehr-/Lernforschung sinnvoll, um eine länderübergreifende Strategie der Entwicklung intelligenter Software $\mathrm{zu}$ erarbeiten. Daran anschließend sollte die öffentliche Förderung von Projektverbünden aus Schule, Wissenschaft und Wirtschaft erfolgen, um intelligente digitale Lernsysteme für die Kernfächer zu entwickeln.

\section{Literaturverzeichnis}

Anger, C. und W. Geis-Thöne (2018), Integration von Kindern und Jugendlichen mit Migrationshintergrund, Herausforderungen für das deutsche Bildungssystem, IW-Analysen Nr. 125.

Anger, C., E. Kohlisch, O. Koppel, A. Plünnecke und R. M. Schüler (2020), MINT-Frühjahrsreport 2020, MINT - Schlüssel für ökonomisches Wohlergehen während der Corona-Krise und nachhaltiges Wachstum in der Zukunft, Gutachten für BDA, BDI, MINT Zukunft schaffen und Gesamtmetall.

Anger, C. und A. Plünnecke (2020a), Homeschooling und Bildungsgerechtigkeit, IW-Kurzbericht Nr. 44/2020.

Anger, C. und A. Plünnecke (2020b), INSM-Bildungsmonitor 2020, Schulische Bildung in Zeiten der Corona-Krise, Gutachten für die INSM.

Anger, S. et al. (2020), Schulschließungen wegen Corona: Regelmäßiger Kontakt zur Schule kann die schulischen Aktivitäten der Jugendlichen erhöhen, IAB-Forum vom 23. April.
Autorengruppe Bildungsberichterstattung (2020), Bildung in Deutschland 2020, Bielefeld, wbv Media.

Belot, M. und D. Webbink (2010), Do teacher strikes harm educational attainment of students?, Labour 24(4), S. 391- 406.

Chetty, R., J. N. Friedman, N. Hendren, M. Stepner and the Opportunity Insights Team (2020), How did COVID-19 and stabilization policies affect spending and employment? A new real-time economic tracker based on private sector data, online verfügbar unter https://opportunityinsights.org/wp-content/uploads/2020/ 05/tracker_paper.pdf.

Di Pietro, G., F. Biagi, P. Costa, Z. Karpinski und J. Mazza (2020), The likely impact of COVID-19 on education: Reflections based on the existing literature and recent international datasets, Publications Office of the European Union, Luxembourg.

Doepke, M. und F. Zilibotti (2019), Love, Money, and Parenting: How Economics Explains the Way We Raise Our Kids, Princeton, Princeton University Press.

Eickelmann, B. et al. (Hrsg.) (2019), ICILS 2018, Computer- und informationsbezogene Kompetenzen von Schülerinnen und Schülern im zweiten internationalen Vergleich und Kompetenzen im Bereich Computational Thinking, Münster, Waxmann Verlag.

Eickelmann, B. und K. Drossel (2020), Schule aufDistanz. Perspektiven und Empfehlungen für den neuen Schulalltag, Befragung des Instituts für Demoskopie Allensbach im Auftrag der Vodafone Stiftung Deutschland, Düsseldorf.

Falck, O., C. Mang und L. Woessmann (2018), Virtually no effect? Different uses of classroom computers and their effect on student achievement, Oxford Bulletin of Economics and Statistics 80(1), S. 1-38.

Gaete, G. (2018), Follow the leader: Student strikes, school absenteeism and persistent consequences on educational outcomes, Working Paper, online verfügbar unter https://papers.ssrn. com/sol3/papers.cfm?abstract_id=2988825.

Geis-Thöne, W. (2020), Häusliches Umfeld in der Krise: Ein Teil der Kinder braucht mehr Unterstützung, Ergebnisse einer Auswertung des Sozio-oekonomischen Panels (SOEP), IW-Report 15/ 2020.

Hachfeld, A., P. Möhrke, S. Schumann und A. Beuter (2020), Lehrerbefragung zur Schulschließung, Erste Ergebnisse Konstanz, online verfügbar unter https://www.bise.uni-konstanz.de/kooperationsnetzwerk-partnerschulen/lehrerbefragung-zur-schulschliessung.

Hattie, J. (2013), The power of feedback in school settings, in R. Sutton (Hrsg.), Feedback: The Handbook of Criticism, Praise, and Advice, New York, Peter Lang Publishing.

Hillmayr, D., F. Reinhold, L. Ziernwald und K. Reiss (2017), Digitale Medien im mathematisch-naturwissenschaftlichen Unterricht der Sekundarstufe; Einsatzmöglichkeiten, Umsetzung und Wirksamkeit, Münster, Waxmann Verlag.

Jaume, D. und A. Willén (2019), The long-run effects of teacher strikes: Evidence from Argentina, Journal of Labor Economics 37(4), S. 1097-139.

Karl, A., D. Entwisle und L. Olson (2007), Lasting consequences of the summer learning gap, American Sociological Review 72(2), S. $167-80$.

Köller, O. (2020), Auswirkungen der Schulschließungen auf die Digitalisierung im Bildungswesen, ifo Schnelldienst 73(9), S. 14-16.

Kuhfeld, M. und B. Tarasawa (2020), The COVID-19 slide: What summer learning loss can tell us about the potential impact of 
school closures on student academic achievement, NWEA Research Brief.

Lavecchia, A. M., P. Oreopoulos und R. S. Brown (2019), Long-run effects from comprehensive student support: Evidence from pathways to education, NBER Working Paper 25630.

Leopoldina (2020), Coronavirus-Pandemie: Für ein krisenresistentes Bildungssystem, 5. Ad-hoc-Stellungnahme vom 5. August 2020.

Liessem, V. (2015), Zahl der Schulabgänger ohne Abschluss bleibt gleich, in Deutscher Caritasverband (Hrsg.), Bildungschancen vor Ort, Studie in Zusammenarbeit mit dem Rheinisch-Westfälischen Institut für Wirtschaftsforschung (RWI).

Maldonado, J.E. und K. De Witte (2020), The effect of school closures on standardised student test outcomes, KU Leuven Discussion Paper DPS20.17.

Montag, C., B. Lachmann, M. Herrlich und K. Zweig (2019), Addictive features of social media/messenger platforms and freemium games against the background of psychological and economic theories, International Journal of Environmental Research and Public Health 16, S. 1-16.

OECD (2020), Were schools equipped to teach - and were students ready to learn - remotely?, PISA in Focus 2020/108.

Ramdass, D. und B. Zimmerman (2011), Developing self-regulation skills: The important role of homework, Journal of Advanced Academics 22(2), S. 194-218.

Wößmann, L. (2020), Folgekosten ausbleibenden Lernens: Was wir über die Corona-bedingten Schulschließungen aus der Forschung lernen können, ifo Schnelldienst 73(6), S. 38-44.

Wößmann, L. et al. (2020), Bildung in der Coronakrise: Wie haben die Schulkinder die Zeit der Schulschließungen verbracht, und welche Bildungsmaßnahmen befürworten die Deutschen?, ifo Schnelldienst 73(9), S. 25-39. 